\title{
LIOUVILLE-TYPE THEOREMS FOR F-HARMONIC MAPS ON NON-COMPACT MANIFOLDS*
}

\author{
JIANCHENG LIU
}

\section{Introduction and main results}

Let $F:[0, \infty) \rightarrow[0, \infty)$ be a $C^{2}$-function such that $F^{\prime}(t)>0$ on $t \in(0, \infty)$. For a smooth map $u:(M, g) \rightarrow(N, h)$ between Riemannian manifolds $(M, g)$ and $(N, h)$. In [1], M. Ara define the $F$-energy $E_{F}(u)$ of $u$ by

$$
E_{F}(u)=\int_{M} F\left(\frac{|d u|^{2}}{2}\right) v_{g},
$$

where $|d u|$ denotes the Hilbert-Schmidt norm of differential $d u \in \Gamma\left(T^{*} M \otimes\right.$ $\left.u^{-1} T N\right)$ with respect to $g$ and $h$, and $v_{g}$ is the volume element of $(M, g)$. We say that $u$ is an $F$-harmonic maps if it is a critical point of the $F$-energy functional.

For example, when $F(t)=t,(2 t)^{p / 2} / p,(1+2 t)^{\alpha}(\alpha>1, m=2)$ and $e^{t}, F$ energy is the energy, the $p$-energy, the $\alpha$-energy of Sacks-Uhlenbeck [2] and the exponential energy respectively. So $F$-harmonic maps is a unified and generalized theory for several varieties of harmonic maps. As a new or more general variational problem suggested by Eells-Sampson [3], it provides many differential geometry interest. Some geometric properties of $F$-harmonic maps, including the first and the second variation formulas, conformal propertites, stability or instability, have been developed in [1], [4] and [5].

In order to represent our interest, we recall that, when $F(t)=t$, i.e. the case of harmonic map, Sampson conjecture that there is no non-constant harmonic map with finite energy from complete simply-connected Riemannian manifolds $M(\operatorname{dim} M \geq 3)$ to any Riemannian manifolds. It is true when $M=\mathbf{R}^{m}$ or $\mathbf{H}^{m}$ proved by Sealey [6]. The same conclusion had obtained by $\mathrm{H}$. S. Hu [7] under the assumption of slowly divergent energy which is the weakening of finite energy. Then Y. L. Xin $[8,9]$ generalized that result to the more general situation, i.e. the starting manifold with "small" negative curvature. More recently, X. Zhang [10] obtain the similar results for $p$-harmonic map (in that case, $\left.F(t)=(2 t)^{p / 2} / p\right)$.

\footnotetext{
* Project supported by Tianyuan Mathematics Fund, NSFC (No. A0324662), the Young Teachers' Scientific Research Fund of Northwest Normal University.

Received April 2, 2004; revised November 11, 2004.
} 
The main purpose in this paper contributes to discuss the nonexistence of non-constant $F$-harmonic map from non-compact manifolds on which some certain restrictions about curvatures are assumed. As a result, we obtain a large classes of Liouville-type theorems for $F$-harmonic maps.

Firstly, we can state one special case of our result without assuming the curvature conditions as follows:

Theorem A. Let $u:\left(\mathbf{R}^{m}, g_{0}\right) \rightarrow\left(N^{n}, h\right)$ be smooth map from $\left(\mathbf{R}^{m}, g_{0}\right)$ into $n$ dimensional Riemannian manifold $N$, where $g_{0}$ is the standard Euclidean metric on $\mathbf{R}^{m}$. Let $F:[0, \infty) \rightarrow[0, \infty)$ be a $C^{2}$-function with $F^{\prime}(t)>0$ and $F(t) \leq t F^{\prime}(t)<$ $\frac{m}{2} F(t)$ on $t \in(0, \infty)$. Suppose that $u$ is $F$-harmonic map with finite $F$-energy, then $u$ is a constant map. A.

In fact, we will prove a theorem which is slight more general than Theorem

THeOREM A'. Let $u:\left(\mathbf{R}^{m}, f g_{0}\right) \rightarrow\left(N^{n}, h\right)$ be smooth map from $\left(\mathbf{R}^{m}, f g_{0}\right)$ into Riemannian manifold $N$, where $g_{0}$ is the standard Euclidean metric and $f$ is a positive smooth function on $\mathbf{R}^{m}$, which satisfies: there exist constant $q<0$ such that

$$
(2-m-q) f(x) \leq \frac{m-2}{2} \frac{\partial f}{\partial x_{i}} \cdot x_{i}
$$

Let $F:[0, \infty) \rightarrow[0, \infty)$ be a $C^{2}$-function with $F^{\prime}(t)>0$ and $F(t) \leq t F^{\prime}(t)<$ $\frac{m}{2} F(t)$ on $t \in(0, \infty)$. Suppose that $u$ is $F$-harmonic map with finite $F$-energy, then $u$ is a constant map.

Remark 1.1. It is easy to see that theorem A is the special case of theorem $\mathrm{A}^{\prime}$ when $f \equiv 1$ on $\mathbf{R}^{m}$.

Remark 1.2. In the cases of harmonic map and $p$-harmonic map, the condition $F(t) \leq t F^{\prime}(t)<\frac{m}{2} F(t)$ say nothing but $m>2$ and $m>p$ respectively. Therefore, theorem $\mathrm{A}^{\prime}$ is an extension of [6] and [11] for harmonic map and $p$ harmonic map.

Secondly, using $F$-stress-energy tensor (defined by M. Ara in [1]), Hessian comparison theorem and Laplace comparison theorem developed by Q. Ding in [12], we obtain the following Liouville-type theorem for $F$-harmonic map.

THEOREM B. Let $M^{m}$ be a m-dimensional $(m>1)$ complete noncompact simply-connected Riemannian manifold, its sectional curvature $K_{M}$ satisfies $-a^{2} \leq$ $K_{M} \leq-b^{2}$, where $a, b$ are some positive constants. Assume that $u: M \rightarrow N$ be $a$ 
$F$-harmonic map with slowly divergent F-energy (see the definition below) from $M$ to any $n$-dimensional Riemannian manifold $N^{n}$. Then $u$ must be a constant map when $(m-1) b F(t)-2 t a F^{\prime}(t) \geq 0$.

Remark 1.3. In the cases of harmonic maps and $p$-harmonic maps, the condition $(m-1) b F(t)-2 t a F^{\prime}(t) \geq 0$ implies that $\frac{m-1}{2} b-a \geq 0$ and $\frac{m-1}{p} b-a \geq 0$ respectively. Therefore, Theorem $\mathrm{B}$ is an extension of [9] and [10] for harmonic maps and $p$-harmonic maps respectively.

THEOREM C. Let $M^{m}$ be a m-dimensional $(m>1)$ complete noncompact simply-connected Riemannian manifold, its sectional curvature $K_{M}$ satisfies $-a^{2} \leq$ $K_{M} \leq 0$, and its Ricci curvature $\operatorname{Ric}_{M} \leq-b^{2}$, where $a$, $b$ are some positive constants. Assume that $u: M \rightarrow N$ be a F-harmonic map with slowly divergent $F$-energy (see the definition below) from $M$ to any $n$-dimensional Riemannian manifold $N^{n}$. Then $u$ must be a constant map when $b F(t)-2 \operatorname{taF}(t) \geq 0$.

Remark 1.4. Theorem $\mathrm{C}$ is also an extension of the result of [9] for harmonic maps, since the condition $b F(t)-2 t a F^{\prime}(t) \geq 0$ implies that $b \geq 2 a$ when $F(t)=t$.

For smooth map $u: M \rightarrow N$, we called the $F$-energy slowly divergent, if there exists certain positive function $\psi(r)$ on $M$ satisfy $\int_{R_{1}}^{\infty} \frac{1}{r \psi(r)} d r=+\infty$,
$\left(R_{1}>0\right)$, such that

$$
\lim _{R \rightarrow \infty} \int_{B_{R}(O)} \frac{F\left(\frac{|d u|^{2}}{2}\right)}{\psi(r(x))} v_{g}<+\infty,
$$

where $r(x)$ be the distance function from fixed point $O \in M$, and $B_{R}(O)$ denotes the geodesic ball of radius $R$ and centered at $O$. In particular, when $\psi(r)$ is a positive constant, (1.3) is nothing but the finiteness of $F$-energy.

\section{Preliminaries and some lemmas}

Let $F:[0, \infty) \rightarrow[0, \infty)$ be a $C^{2}$-function such that $F^{\prime}>0$ on $(0, \infty)$. Let $u: M \rightarrow N$ be a smooth map from an $m$-dimensional Riemannian manifold $(M, g)$ to a Riemannian manifold $(N, h)$. We call $u$ an $F$-harmonic map if it is a critical point of $F$-energy functional. That is, $u$ is an $F$-harmonic map if and only if

$$
\left.\frac{d}{d t} E_{F}\left(u_{t}\right)\right|_{t=0}=0
$$

for any compactly supported variation $u_{t}: M \rightarrow N(-\varepsilon<t<\varepsilon)$ with $u_{0}=u$. 
Let $\nabla$ and ${ }^{N} \nabla$ always denote the Levi-Civita connections of $M$ and $N$ respectively. Let $\tilde{\nabla}$ be the induced connection on $u^{-1} T N$ defined by $\tilde{\nabla}_{X} W=$ ${ }^{N} \nabla_{u_{*} X} W$, where $X$ is a tangent vector of $M$ and $W$ is a section of $u^{-1} T N$. We choose a local orthonormal frame field $\left\{e_{i}\right\}_{i=1}^{m}$ on $M$, then the $F$-tension field $\tau_{F}(u)$ of $u$ defined by (cf. [1])

$$
\begin{aligned}
\tau_{F}(u) & =\sum_{i=1}^{m}\left\{\tilde{\nabla}_{e_{i}}\left(F^{\prime}\left(\frac{|d u|^{2}}{2}\right) u_{*} e_{i}\right)-F^{\prime}\left(\frac{|d u|^{2}}{2}\right) u_{*} \nabla_{e_{i}} e_{i}\right\} \\
& =F^{\prime}\left(\frac{|d u|^{2}}{2}\right) \tau(u)+u_{*}\left\{\operatorname{grad}\left(F^{\prime}\left(\frac{|d u|^{2}}{2}\right)\right)\right\},
\end{aligned}
$$

where $\tau(u)=\sum_{i=1}^{m}\left(\tilde{\nabla}_{e_{i}} u_{*} e_{i}-u_{*} \nabla_{e_{i}} e_{i}\right)$ is the tension field of $u$.

Under the notation above, following from [1], the first variation formula for $F$-harmonic map reads as

$$
\left.\frac{d}{d t} E_{F}\left(u_{t}\right)\right|_{t=0}=-\int_{M} h\left(V, \tau_{F}(u)\right) v_{g}
$$

where $V=\left.\frac{d u_{t}}{d t}\right|_{t=0}$. Therefore a smooth map $u: M \rightarrow N$ is an $F$-harmonic map if and only if the $F$-tension field $\tau_{F}(u)=0$. Many examples of $F$-harmonic map are given in [1].

For the smooth map $u:\left(M^{m}, g\right) \rightarrow\left(N^{n}, h\right)$, M. Ara introduces in [1] the stress energy tensor $S_{F}(u)$ of $u$ associated to the $F$-energy functional $E_{F}$ (which we call, the $F$-stress energy tensor of $u$, in short) is given by

$$
S_{F}(u)=F\left(\frac{|d u|^{2}}{2}\right) \cdot g-F^{\prime}\left(\frac{|d u|^{2}}{2}\right) \cdot u^{*} h .
$$

For any vector field $X$ on $M$, the relation between $F$-tension field and $F$ stress energy tensor can be written as

$$
\left(\operatorname{div} S_{F}(u)\right)(X)=-h\left(\tau_{F}(u), u_{*} X\right) .
$$

Lemma 1. Let $D \subseteq M$ be a compact domain, its boundary $\partial D$ be a smooth hypersurface in $M$. Then, for any $C^{2}$-map $u:(M, g) \rightarrow(N, h)$ and any smooth vector field $X$ on $M$, we have

$$
\begin{aligned}
\int_{\partial D} F\left(\frac{|d u|^{2}}{2}\right) g(X, \boldsymbol{n}) v_{g}= & \int_{\partial D} F^{\prime}\left(\frac{|d u|^{2}}{2}\right) h\left(u_{*} X, u_{*} \boldsymbol{n}\right) v_{g}+\int_{D}\left(\operatorname{div} S_{F}(u)\right)(X) v_{g} \\
& +\int_{D}\left\langle S_{F}(u), \nabla X\right\rangle v_{g}
\end{aligned}
$$

where $\boldsymbol{n}$ be the unit normal vector of $\partial D$. 
Proof. Choosing a local orthonormal frame field $\left\{e_{i}\right\}_{i=1}^{m}$ on $M$, and define $\nabla X\left(e_{i}, e_{j}\right):=\left\langle\nabla_{e_{i}} X, e_{j}\right\rangle$, then

$$
\begin{aligned}
\nabla_{X} F & \left(\frac{|d u|^{2}}{2}\right) \\
& =F^{\prime}\left(\frac{|d u|^{2}}{2}\right) \nabla_{X}\left(\frac{|d u|^{2}}{2}\right) \\
& =F^{\prime}\left(\frac{|d u|^{2}}{2}\right)\left\{\operatorname{div}\left(h\left(u_{*} X, u_{*} e_{i}\right) e_{i}\right)-h\left(u_{*} X, \tau(u)\right)-\left\langle\nabla X, u^{*} h\right\rangle\right\} \\
& =\operatorname{div}\left(F^{\prime}\left(\frac{|d u|^{2}}{2}\right) h\left(u_{*} X, u_{*} e_{i}\right) e_{i}\right)-h\left(u_{*} X, \tau_{F}(u)\right)-\left\langle\nabla X, F^{\prime}\left(\frac{|d u|^{2}}{2}\right) \cdot u^{*} h\right\rangle
\end{aligned}
$$

Therefore

$$
\begin{aligned}
& \operatorname{div}\left(F\left(\frac{|d u|^{2}}{2}\right) X\right) \\
& =\left(\nabla_{e_{i}} F\left(\frac{|d u|^{2}}{2}\right)\right) g\left(X, e_{i}\right)+F^{\prime}\left(\frac{|d u|^{2}}{2}\right) g\left(\nabla_{e_{i}} X, e_{i}\right) \\
& =\nabla_{X} F\left(\frac{|d u|^{2}}{2}\right)+F\left(\frac{|d u|^{2}}{2}\right)\langle\nabla X, g\rangle \\
& =\operatorname{div}\left(F^{\prime}\left(\frac{|d u|^{2}}{2}\right) h\left(u_{*} X, u_{*} e_{i}\right) e_{i}\right)-h\left(u_{*} X, \tau_{F}(u)\right)+\left\langle\nabla X, S_{F}(u)\right\rangle
\end{aligned}
$$

Now, for compact domain $D$ in $M$, taking local orthonormal frame field $\left\{e_{i}\right\}_{i=1}^{m}$ on $M$ along $\partial D$, such that $e_{1}, \ldots, e_{m-1} \in \Gamma(T \partial D)$, and $e_{m}=\boldsymbol{n}$ be the unit normal vector of $\partial D$. Integrating (2.5) on $D$, by means of Green's theorem and (2.3), we complete the proof of Lemma 1.

\section{The Proof of Theorem $\mathrm{A}$ and $\mathbf{A}^{\prime}$}

Define a family $\left\{V_{t}\right\}_{t \in \mathbf{R}^{+}}$of maps $V_{t}: \mathbf{R}^{m} \rightarrow N$ by $V_{t}(x)=u(t x)$ for $x \in \mathbf{R}^{m}$, and set

$$
\Phi(R, t)=\int_{B(R)} F\left(\frac{\left|d V_{t}\right|^{2}}{2}\right) v_{g},
$$

where $B(R)=\left\{x \in \mathbf{R}^{m}|| x \mid<R\right\}$. Applying Green's theorem, we have 


$$
\begin{aligned}
\left.\frac{\partial}{\partial t} \Phi(R, t)\right|_{t=1}= & \left.\int_{B(R)} F^{\prime}\left(\frac{\left|d V_{t}\right|^{2}}{2}\right)\left\langle d V_{t}, \frac{d}{d t}\left(d V_{t}\right)\right\rangle\right|_{t=1} v_{g} \\
= & \int_{B(R)}\left\langle F^{\prime}\left(\frac{|d u|^{2}}{2}\right) d u, \tilde{\nabla}\left(d u\left(r \frac{\partial}{\partial r}\right)\right)\right\rangle v_{g} \\
= & \int_{B(R)}\left\langle d^{*}\left(F^{\prime}\left(\frac{|d u|^{2}}{2}\right) d u\right), d u\left(r \frac{\partial}{\partial r}\right)\right\rangle v_{g} \\
& +R \int_{\partial B(R)} F^{\prime}\left(\frac{|d u|^{2}}{2}\right)\left\langle d u\left(\frac{\partial}{\partial \boldsymbol{n}}\right), d u\left(r \frac{\partial}{\partial r}\right)\right\rangle \sigma_{R}
\end{aligned}
$$

where $\frac{\partial}{\partial \boldsymbol{n}}=f^{-1} \frac{\partial}{\partial r}$ is the unit normal and $\sigma_{R}$ denotes the volume element of the induced Riemannian metric on $\partial B(R)$. By the $F$-harmonic condition $d^{*}\left(F\left(\frac{|d u|^{2}}{2}\right) d u\right)=0$ and $d u\left(\frac{\partial}{\partial \boldsymbol{n}}\right)=f^{-1} d u\left(\frac{\partial}{\partial r}\right)$, it follows that

$$
\left.\frac{\partial}{\partial t} \Phi(R, t)\right|_{t=1} \geq 0
$$

On the other hand, reparameterizing the integral (3.1), we get

$$
\Phi(R, t)=t^{-m} \int_{B(t R)} F\left(\frac{1}{2} t^{2} f^{-1}\left(\frac{x}{t}\right) h_{k l}(u(x)) \frac{\partial u^{k}(x)}{\partial x_{i}} \frac{\partial u^{l}(x)}{\partial x_{i}}\right) f^{m / 2}\left(\frac{x}{t}\right) d x .
$$

By a direct calculation, we have

$$
\begin{aligned}
& \frac{\partial}{\partial t} \Phi(R, t) \\
& =(-m) t^{m-1} \int_{B(t R)} F\left(\frac{1}{2} t^{2} f^{-1}\left(\frac{x}{t}\right) h_{k l}(u(x)) \frac{\partial u^{k}(x)}{\partial x_{i}} \frac{\partial u^{l}(x)}{\partial x_{i}}\right) f^{m / 2}\left(\frac{x}{t}\right) d x \\
& \quad-\frac{m}{2} t^{-m} \int_{B(t R)} F\left(\frac{1}{2} t^{2} f^{-1}\left(\frac{x}{t}\right) h_{k l}(u(x)) \frac{\partial u^{k}(x)}{\partial x_{i}} \frac{\partial u^{l}(x)}{\partial x_{i}}\right) f^{(m-2) / 2}\left(\frac{x}{t}\right)\left(\frac{x_{i}}{t^{2}} \frac{\partial f}{\partial x_{i}}\right) d x \\
& \quad+t^{-m} \int_{\partial B(t R)} R(R t)^{m-2} F\left(\frac{1}{2} t^{2} f^{-1}\left(\frac{x}{t}\right) h_{k l}(u(x)) \frac{\partial u^{k}(x)}{\partial x_{i}} \frac{\partial u^{l}(x)}{\partial x_{i}}\right) f^{m / 2}\left(\frac{x}{t}\right) \sigma_{R} \\
& \quad+t^{-m} \int_{B(t R)} F^{\prime}\left(\frac{1}{2} t^{2} f^{-1}\left(\frac{x}{t}\right) h_{k l}(u(x)) \frac{\partial u^{k}(x)}{\partial x_{i}} \frac{\partial u^{l}(x)}{\partial x_{i}}\right) f^{m / 2}\left(\frac{x}{t}\right) \\
& \quad \times h_{k l}(u(x)) \frac{\partial u^{k}(x)}{\partial x_{i}} \frac{\partial u^{l}(x)}{\partial x_{i}}\left\{t f^{-1}\left(\frac{x}{t}\right)+\frac{1}{2} t^{2} f^{-2}\left(\frac{x}{t}\right)\left(\frac{x_{i}}{t^{2}} \frac{\partial f}{\partial x_{i}}\right)\right\} d x .
\end{aligned}
$$


At $t=1$, we obtain from $(3.5)$

$$
\begin{aligned}
& \left.\frac{\partial}{\partial t} \Phi(R, t)\right|_{t=1} \\
& =\int_{B(R)}\left\{2 F^{\prime}\left(\frac{|d u|^{2}}{2}\right) \cdot \frac{|d u|^{2}}{2}-m F\left(\frac{|d u|^{2}}{2}\right)\right\} f^{m / 2}(x) d x \\
& \quad+\int_{B(R)}\left\{F^{\prime}\left(\frac{|d u|^{2}}{2}\right) \cdot \frac{|d u|^{2}}{2}-\frac{m}{2} F\left(\frac{|d u|^{2}}{2}\right)\right\} f^{(m-2) / 2}(x)\left(x_{i} \frac{\partial f}{\partial x_{i}}\right) d x \\
& \quad+R \int_{\partial B(R)} R^{m-2} F\left(\frac{|d u|^{2}}{2}\right) f^{m / 2}(x) \sigma_{R}
\end{aligned}
$$

If $F(t) \leq t F^{\prime}(t)<\frac{m}{2} F(t)$, combining (1.2) and (3.6), we get

$$
\begin{aligned}
\left.\frac{\partial}{\partial t} \Phi(R, t)\right|_{t=1} \leq & q \int_{B(R)} F\left(\frac{|d u|^{2}}{2}\right) f^{m / 2}(x) d x \\
& +R \int_{\partial B(R)} R^{m-2} F\left(\frac{|d u|^{2}}{2}\right) f^{m / 2}(x) \sigma_{R} \\
= & q \Phi(R, 1)+R \frac{d}{d R} \Phi(R, 1) .
\end{aligned}
$$
From (3.3) and (3.7), we have $q \Phi(R, 1)+R \frac{d}{d R} \Phi(R, 1) \geq 0$. Therefore, for all
$R>0$, it follows that

$$
\frac{d}{d R}\left\{R^{q} \Phi(R, 1)\right\} \geq 0
$$

Now, suppose that $u$ is a nonconstant $F$-harmonic map, by the continuation property, $|d u|^{2}$ cannot vanish identically on some open set in $\mathbf{R}^{m}$. Thus there exists $R_{0}>0$ and $C>0$, such that $\int_{B\left(R_{0}\right)} F\left(\frac{|d u|^{2}}{2}\right) v_{g} \geq C$, meanwhile, for all
$R \geq R_{0}$, we have

$$
\int_{B(R)} F\left(\frac{|d u|^{2}}{2}\right) v_{g} \geq\left(\frac{R_{0}}{R}\right)^{q} \int_{B\left(R_{0}\right)} F\left(\frac{|d u|^{2}}{2}\right) v_{g} \geq C\left(\frac{R_{0}}{R}\right)^{q},
$$

since $q<0$, hence

$$
E_{F}(u)=\lim _{R \rightarrow \infty} \int_{B(R)} F\left(\frac{|d u|^{2}}{2}\right) v_{g} \geq \infty
$$


which gives a contradiction to the finiteness condition of $E_{F}(u)$. We complete the proof of theorem $\mathrm{A}^{\prime}$ and theorem $\mathrm{A}$ as a corollary of theorem $\mathrm{A}^{\prime}$.

\section{The Proof of Theorem $B$ and $C$}

Proof of Theorem B. Denoted by $D=B_{R}\left(x_{0}\right)$ the geodesic ball of radius $R$ and centered at $x_{0} \in M$. Taking $X=r \frac{\partial}{\partial r} \in T_{x_{0}} M\left(\frac{\partial}{\partial r}\right.$ denoted unit radial vector field and $r=r(x)$ denoted the distance function from $\left.x_{0}\right)$. Choosing a local orthonormal frame field $\left\{e_{1}, \ldots, e_{m-1}, \frac{\partial}{\partial r}\right\}$ on $M$. After applying $D=B_{R}\left(x_{0}\right)$ and $X=r \frac{\partial}{\partial r}$ to $(2.4)$, we have

$$
\begin{aligned}
\int_{B_{R}\left(x_{0}\right)} & \left(\operatorname{div} S_{F}(u)\right)(X) v_{g}+\int_{B_{R}\left(x_{0}\right)}\left\langle S_{F}(u), \nabla X\right\rangle v_{g} \\
= & \int_{\partial B_{R}\left(x_{0}\right)} F\left(\frac{|d u|^{2}}{2}\right) g(X, \boldsymbol{n}) v_{g}-\int_{\partial B_{R}\left(x_{0}\right)} F\left(\frac{|d u|^{2}}{2}\right) h\left(u_{*} X, u_{*} n\right) v_{g} \\
= & R \int_{\partial B_{R}\left(x_{0}\right)} F\left(\frac{|d u|^{2}}{2}\right) v_{g}-R \int_{\partial B_{R}\left(x_{0}\right)} F\left(\frac{|d u|^{2}}{2}\right) h\left(u_{*} \frac{\partial}{\partial r}, u_{*} \frac{\partial}{\partial r}\right) v_{g} \\
\leq & R \int_{\partial B_{R}\left(x_{0}\right)} F\left(\frac{|d u|^{2}}{2}\right) v_{g} .
\end{aligned}
$$

Now, we will compute the item $\left\langle S_{F}(u), \nabla X\right\rangle$ on the left hand side of (4.1). For this purpose, using local orthonormal frame field $\left\{e_{1}, \ldots, e_{m-1}, \frac{\partial}{\partial r}\right\}$, it is easy
to see that

$$
\begin{gathered}
\nabla_{\partial / \partial r} X=\frac{\partial}{\partial r}, \quad \nabla_{e_{i}} X=r \nabla_{e_{i}} \frac{\partial}{\partial r}=r \operatorname{Hess}(r)\left(e_{i}, e_{j}\right) e_{j}, \\
\operatorname{div} X=1+r \operatorname{Hess}(r)\left(e_{i}, e_{i}\right), \quad 1 \leq i \leq m-1,
\end{gathered}
$$

where Hess $(\cdot)$ denoted the Hessian operator, i.e. Hess $(r)\left(e_{i}, e_{j}\right)=\nabla_{e_{j}} \nabla_{e_{i}} r-\left(\nabla_{e_{j}} e_{i}\right) r$. So

$$
\begin{aligned}
& F\left(\frac{|d u|^{2}}{2}\right) h\left(u_{*} e_{\alpha}, u_{*} e_{\beta}\right) \cdot g\left(\nabla_{e_{\alpha}} X, e_{\beta}\right) \\
& \quad=F\left(\frac{|d u|^{2}}{2}\right)\left\{r \operatorname{Hess}\left(e_{i}, e_{j}\right) h\left(u_{*} e_{i}, u_{*} e_{j}\right)+h\left(u_{*} \frac{\partial}{\partial r}, u_{*} \frac{\partial}{\partial r}\right)\right\} .
\end{aligned}
$$

Then 


$$
\begin{aligned}
\left\langle S_{F}(u), \nabla X\right\rangle= & F\left(\frac{|d u|^{2}}{2}\right) \operatorname{div} X-F^{\prime}\left(\frac{|d u|^{2}}{2}\right) h\left(u_{*} e_{\alpha}, u_{*} e_{\beta}\right) \cdot g\left(\nabla_{e_{\alpha}} X, e_{\beta}\right) \\
= & F\left(\frac{|d u|^{2}}{2}\right)\left(1+r \operatorname{Hess}(r)\left(e_{i}, e_{i}\right)\right) \\
& -F^{\prime}\left(\frac{|d u|^{2}}{2}\right)\left\{\left|u_{*} \frac{\partial}{\partial r}\right|^{2}+r \operatorname{Hess}(r)\left(e_{i}, e_{j}\right) h\left(u_{*} e_{i}, u_{*} e_{j}\right)\right\} .
\end{aligned}
$$

If the sectional curvature $K_{M}$ of $M$ satisfy the condition in Theorem B, applying Hessian comparison theorem (cf. [9]), we compute directly and get

$$
\begin{aligned}
&\left\langle S_{F}(u), \nabla X\right\rangle \\
& \geq F\left(\frac{|d u|^{2}}{2}\right)\{1+(m-1)(b r) \operatorname{coth}(b r)\} \\
&-F^{\prime}\left(\frac{|d u|^{2}}{2}\right)\left\{\left|u_{*} \frac{\partial}{\partial r}\right|^{2}+(a r) \operatorname{coth}(a r) h\left(u_{*} e_{i}, u_{*} e_{i}\right)\right\} \\
& \geq F\left(\frac{|d u|^{2}}{2}\right)\{1+(m-1)(b r) \operatorname{coth}(b r)\} \\
&-F^{\prime}\left(\frac{|d u|^{2}}{2}\right)\left\{(a r) \operatorname{coth}(a r)\left|u_{*} \frac{\partial}{\partial r}\right|^{2}+(a r) \operatorname{coth}(a r) h\left(u_{*} e_{i}, u_{*} e_{i}\right)\right\} \\
&= F\left(\frac{|d u|^{2}}{2}\right)\{1+(m-1)(b r) \operatorname{coth}(b r)\}-F^{\prime}\left(\frac{|d u|^{2}}{2}\right)(a r) \operatorname{coth}(a r)|d u|^{2} \\
& \geq F\left(\frac{|d u|^{2}}{2}\right)+r \cdot \operatorname{coth}(b r)\left\{(m-1) b \cdot F\left(\frac{|d u|^{2}}{2}\right)-a|d u|^{2} F^{\prime}\left(\frac{|d u|^{2}}{2}\right)\right\} .
\end{aligned}
$$

Hence, when $(m-1) b F(t)-2 t a F^{\prime}(t) \geq 0$, it follows from (4.2)

$$
\left\langle S_{F}(u), \nabla X\right\rangle \geq F\left(\frac{|d u|^{2}}{2}\right) .
$$

According to (2.3), (4.1) and (4.3), for $F$-harmonic map $u$, we obtain

$$
R \int_{\partial B_{R}\left(x_{0}\right)} F\left(\frac{|d u|^{2}}{2}\right) v_{g} \geq \int_{B_{R}\left(x_{0}\right)} F\left(\frac{|d u|^{2}}{2}\right) v_{g} .
$$

Suppose that $u:\left(M^{m}, g\right) \rightarrow\left(N^{n}, h\right)$ is a non-constant $F$-harmonic map, i.e. $|d u|^{2} \neq 0$ at some point of $x \in M$, then there exists $R_{0}>0$, such that, when $R>R_{0}$,

$$
\int_{B_{R}\left(x_{0}\right)} F\left(\frac{|d u|^{2}}{2}\right) v_{g} \geq C_{0},
$$


where $C_{0}$ be a positive constant. From (4.4), then

$$
\int_{\partial B_{R}\left(x_{0}\right)} F\left(\frac{|d u|^{2}}{2}\right) v_{g} \geq \frac{C_{0}}{R} .
$$

Since the $F$-energy of $u$ divergent slowly, therefore, (4.5) will imply

$$
\begin{aligned}
\lim _{R \rightarrow \infty} \int_{B_{R}\left(x_{0}\right)} \frac{F\left(\frac{|d u|^{2}}{2}\right)(x)}{\psi(r(x))} v_{g} & =\int_{0}^{\infty} \frac{d R}{\psi(R)} \int_{\partial B_{R}\left(x_{0}\right)} F\left(\frac{|d u|^{2}}{2}\right) v_{g} \\
& \geq C_{0} \int_{0}^{\infty} \frac{d R}{R \psi(R)} \\
& \geq C_{0} \int_{R_{0}}^{\infty} \frac{d R}{R \psi(R)}=\infty .
\end{aligned}
$$

That's in contradiction with $F$-energy of $u$ being slowly divergent. So $u$ must be a constant map. We complete the proof of Theorem B.

Proof of Theorem C. We will continue using the symbol in the proof of theorem B. Taking another form of divergence for $X=r \frac{\partial}{\partial r}$, i.e. $\operatorname{div} X=$ $1+r \Delta r$. Since $\operatorname{Ric}_{M} \leq-b^{2}$, the Laplace comparison theorem (cf. [12]) due to Q. Ding says

then

$$
\Delta r \geq b \cdot \operatorname{coth}(b r)
$$

$$
\begin{aligned}
&\left\langle S_{F}(u), \nabla X\right\rangle \\
& \geq F\left(\frac{|d u|^{2}}{2}\right)\{1+(b r) \operatorname{coth}(b r)\} \\
&-F^{\prime}\left(\frac{|d u|^{2}}{2}\right)\left\{\left|u_{*} \frac{\partial}{\partial r}\right|^{2}+(a r) \operatorname{coth}(a r) h\left(u_{*} e_{i}, u_{*} e_{i}\right)\right\} \\
& \geq F\left(\frac{|d u|^{2}}{2}\right)\{1+(b r) \operatorname{coth}(b r)\} \\
&-F^{\prime}\left(\frac{|d u|^{2}}{2}\right)\left\{(a r) \operatorname{coth}(a r)\left|u_{*} \frac{\partial}{\partial r}\right|^{2}+(a r) \operatorname{coth}(a r) h\left(u_{*} e_{i}, u_{*} e_{i}\right)\right\} \\
&= F\left(\frac{|d u|^{2}}{2}\right)\{1+(b r) \operatorname{coth}(b r)\}-F^{\prime}\left(\frac{|d u|^{2}}{2}\right)(a r) \operatorname{coth}(a r)|d u|^{2} \\
& \geq F\left(\frac{|d u|^{2}}{2}\right)+r \cdot \operatorname{coth}(b r)\left\{b \cdot F\left(\frac{|d u|^{2}}{2}\right)-a|d u|^{2} F^{\prime}\left(\frac{|d u|^{2}}{2}\right)\right\} .
\end{aligned}
$$


Meanwhile, when $b F(t)-2 t a F^{\prime}(t) \geq 0$, (4.6) becomes

$$
\left\langle S_{F}(u), \nabla X\right\rangle \geq F\left(\frac{|d u|^{2}}{2}\right) .
$$

For $F$-harmonic map $u$, applying $D=B_{R}\left(x_{0}\right)$ and $X=r \frac{\partial}{\partial r}$ to (2.4), combining (2.3) with (4.7), by proceeding similarly as in the proof of theorem B, we proved that $u$ must be a constant map.

Acknowledgements. The author would like to thank Professor Shen Chunli and Professor $\mathrm{Yu}$ Yanlin for helpful comments concerning this paper. He would also like to thank Liao Caisheng for hospitality.

\title{
REFERENCES
}

[1] M. Ara, Geometry of F-Harmonic Maps, Kodai Math. J. 22 (1999), 243-263.

[2] J. Sacks and K. Uhlenbeck, The existence of minimal immersions of 2-spheres, Ann. of Math. 113 (1981), 1-24.

[ 3 ] J. Eells and J. H. SAmpson, Harmonic mappings of Riemannian manifolds, Amer. J. Math. 86 (1964), 109-160.

[4] M. ARA, Instability and nonexistence theorems for F-Harmonic Maps, Illinois J. Math. 45 (2001), 657-680.

[5] J. T. LI, F-Harmonic maps for positively curved manifolds (in Chinese), Acta Math. Sinica 46 (2003), 811-814.

[6] H. C. J. Sealey, Some conditions ensuring the vanishing of harmonic differential forms with applications to harmonic maps and Yang-Mills theory, Math. Proc. Camb. Phil. Soc. 91 (1982), 441-452.

[7] H. S. Hu, An nonexistence theorem for harmonic maps with slowly divergent energy, Chinese Ann. Math., Ser. B. 5 (1984), 737-740.

[8] Y. L. XIN, Liouville type theorems and regularity of harmonic maps, Lecture Notes Math. 1255, Springer-Verlag, 1987, 198-208.

[9] Y. L. XIN, Harmonic Maps (in Chinese), Shanghai Scientific and Technical Publishers, Shanghai, 1995.

[10] X. Zhang, Liouville Theorems for $p$-Harmonic maps (in Chinese), Chinese Annals of Mathematics, Ser. A. 21 (2000), 95-98.

[11] H. TAKeUchi, Stability and Liouville Theorems of $p$-Harmonic maps, Janan J. Math., New Ser. 17 (1991), 317-332.

[12] Q. Ding, The Dirichlet problem at infinity for manifolds of nonpositive curvature, Proc. Symp. Diff. Geom. in Honour of Professor Su Buchin on his 90th Birthday, World Sci. Publ. Co., 1993, 49-58.

\author{
Department of Mathematics \\ NorthWEST NoRmal UNIVERSITY \\ LANZHOU 730070 \\ CHINA \\ E-mail: liujc@nwnu.edu.cn
}

